\title{
The transcondylar approach to craniocervical meningiomas
}

\author{
Marcio S. Rassi, MD, ${ }^{1}$ Jean G. de Oliveira, MD, PhD, ${ }^{2}$ and Luis A. B. Borba, MD, $\mathrm{PhD}^{1,3}$ \\ ${ }^{1}$ Department of Neurosurgery, Evangelic University Hospital of Curitiba, Parana; ${ }^{2 D i v i s i o n ~ o f ~ C e r e b r o v a s c u l a r ~ a n d ~ S k u l l ~ B a s e ~}$ \\ Surgery, Center of Neurology and Neurosurgery Associates (CENNA), Hospital Beneficência Portuguesa de São Paulo; and \\ ${ }^{3}$ Department of Neurosurgery, Federal University of Parana, Curitiba, Parana, Brazil
}

Surgical removal of foramen magnum meningiomas poses great challenges due their deep location within the central skull base and their proximity to vital neurovascular structures. This video depicts the operative nuances of surgical management for a 59-year-old female who presented with a right-sided spinocranial meningioma. Simpson Grade I resection was achieved through a right transcondylar approach. The patient's postoperative period was unremarkable, and she was discharged home on postoperative Day 5 for periodic follow-up. The transcondylar approach safely exposes the craniocervical junction at the anterior aspect of the neuraxis and still allows the surgeon to access the tumor through a parallel plane, with minimum morbidity.

The video can be found here: https://youtu.be/P0-kXjAkw9U.

KEYWORDS meningioma; foramen magnum; transcondylar; skull base; brain tumor 